\title{
Allergy immunotherapies for allergic rhinitis: systematic review and assessment of evolving quality
}

\author{
${ }^{1}$ ALK-Abelló, Hørsholm, Denmark \\ ${ }^{2}$ RTI Health Solutions, Manchester, United Kingdom
}

\author{
KEY WORDS \\ allergic asthma; allergic rhinitis; allergy \\ immunotherapy; grass allergy; quality assessment
}

\section{Corresponding author}

Annete Njue

RTI Health Solutions

The Pavilion, Towers Business Park

Wilmslow Road, Didsbury, Manchester

M20 2LS, United Kingdom

E-mail: anjue@rti.org

Phone: +441614476034

Fax: +441614348232

Doi

10.23822/EurAnnACI.1764-1489.100

\begin{abstract}
Summary
Background. Heterogeneity in the design and quality of trials evaluating allergy immunotherapies (AITs) limits their comparability, making it difficult for physicians, patients, and payers to select the best treatment option. Methods. This systematic review evaluated the quality of randomised controlled trials (RCTs) of registered grass AITs using the National Institute of Health and Care Excellence checklist. Results. 17 of 44 unique RCTs (38.6\%) (sample size range: 18-1,501 subjects) were subcutaneous grass immunotherapy trials and 27 (61.4\%) were sublingual grass immunotherapy trials (Allergovit, 5 trials; Alutard, 8; Grazax, 13; Oralair, 6; Staloral, 8; Pollinex, 2; Phostal and Purethal, 1 each). Three trials (6.8\%; all Grazax) fulfilled every quality criterion. Quality assessments revealed inconsistencies in study quality and reporting. Study quality trended towards improvement over time, particularly after 2009. Conclusions. When assessing grass AIT, it is important to focus not only on endpoints but also on the quality of evidence.
\end{abstract}

\section{Introduction}

Allergic rhinitis/allergic rhinitis with conjunctivitis (AR/ARC) and asthma are considered separate manifestations of the same disease: chronic airway inflammation, occurring in the upper airway in AR/ARC and in the lower airway in asthma $(1,2)$. $\mathrm{AR} / \mathrm{ARC}$ is one of the most important risk factors for asthma and typically precedes the development of asthma, contributing to unsatisfactory disease control (2-4). Early diagnosis and treatment of AR/ARC is crucial to halt the progression of the disease to asthma $(3,5)$. Symptom-relieving pharmacotherapy for AR/ARC is not effective for all patients and does not prevent development of asthma symptoms because it does not treat the underlying disease (6). Allergy immunotherapy (AIT), or gradual exposure to an allergen to desensitise the immune response to trigger allergen, is a treatment option for patients with AR/ARC related to grass pollen and other allergens whose symptoms are inadequately controlled by pharmacotherapy. Allergy immunotherapy treats the underlying disease, thereby reducing symptoms $(1,7,8)$.

Selection of the most appropriate AIT treatment for individuals with AR/ARC is complex, in part because of the historical background of the development of AIT. Historically, allergen extracts have not been seen as medical products in the European market, and named patient products, which may be distributed in European countries without a marketing authorisation (9), have been and continue to be widely used $(10,11)$. Moreover, the first regulatory approvals were granted to AIT products with very limited or even no randomised controlled trials (RCTs) supporting a positive benefit-risk profile. For physicians and patients who decide to use a registered AIT product, the challenge is to find a product with well-documented evidence for the efficacy and safety. 
Allergen-specific AITs for AR/ARC may be administered subcutaneously or sublingually. In European countries, subcutaneous immunotherapy (SCIT) has been available for more than a century (12). Sublingual immunotherapy (SLIT), which is available in drop formulation and, more recently, in tablet formulation, was first licensed in 2006 (13). There are limitations in the evidence base for SCIT and SLIT products. Early trials of AITs were often uncontrolled and included small sample sizes (10). Placebo-controlled clinical trials are now common, and the quality of these trials has improved in the past decade (14). Nevertheless, heterogeneity in trial design and population limits the comparability of trial results $(10,11,14)$. The European Academy of Allergy and Clinical Immunology (EAACI) has published recommendations regarding clinical outcomes in AIT trials for ARC. These are likely to assist in standardising outcome measures to enable better analysis of clinical efficacy and improve the comparability of results (15).

Clinical guidelines recommend AIT for uncontrolled AR/ARC symptoms. The Allergic Rhinitis and Its Impact on Asthma (ARIA) clinical guidelines recommend AIT, including SLIT or intranasal allergen-specific immunotherapy, for the treatment of AR due to pollen (16). In 2017, the EAACI issued guidelines for AIT for the treatment of AR (17). These guidelines note that some AIT products do not provide sufficient data to support their efficacy in clinical practice and recommend that only standardised AIT products with documentation of efficacy should be prescribed. Specifically, the guidelines recommend preseasonal/co-seasonal SLIT for seasonal AR for short-term benefit and grass pollen SLIT tablets or solution with continuous therapy for AR for long-term benefit.

To support treatment decision making for AITs for AR/ARC, the objective of this study was to conduct a systematic literature review to identify placebo-controlled RCTs of grass AITs used for the treatment of patients with AR/ARC, with the aim of evaluating the quality of published evidence. The review was restricted to grass AIT products that are registered in Europe, including Allergovit, Alutard/ALK Depot, ALK start, Grazax, Oralair, Phostal, Pollinex, Polvac, Purethal, and Staloral.

\section{Materials and methods}

Searches were performed on the MEDLINE, Embase, Biosciences Information Service (BIOSIS), and Cochrane Library electronic literature databases on 25 January 2017, with no date, language, or geographical restrictions. Updated searches of the same databases were performed on 24 April 2018. In addition, conference abstracts (EAACI; American Academy of Allergy, Asthma and Immunology [AAAAI]; European Respiratory Society [ERS]; American Thoracic Society [ATS]) were searched from 1 January 2015 to 30 December 2016. Two study registries (ClinicalTrials.gov and the European Union Clinical Trials Register [https://www.clinicaltrialsregister. $\mathrm{eu} /]$ ) were also searched for completed trials with results. Bibliographic lists of included recent relevant systematic literature reviews and meta-analyses were searched for further studies of interest.

Search terms included combinations of free text and Medical Subject Headings (MeSH). Specifically, the searches included terms for the population of interest (disease), including AR or ARC and grass or grass pollen (e.g., ("Rhinitis, Allergic" $[\mathrm{MeSH}] \mathrm{OR}$ "allergic rhinitis" [Text Word] OR "allergic rhinoconjunctivitis"[Text Word]) AND grass[Text Word]); for the interventions or comparators of interest (e.g., "allergy immunotherapy" [Text Word] OR "sublingual immunotherapy"[Text Word]); and for the study types of interest, including placebo-controlled, randomised, clinical trials (e.g., "Randomized Controlled Trials as Topic" [MeSH] OR "Randomized Controlled Trial"[Publication Type]). Animal studies, phase 1 trials, comments, and editorials were excluded.

The study selection process occurred in 2 phases, during which studies were screened for relevance based on study design, population, interventions included, and language of publication. Table I presents the inclusion and exclusion criteria that were used at the level 1 and level 2 screenings. Specifically, at level 1 screening, titles and abstracts of identified studies were reviewed independently by 2 researchers (double screening) for eligibility according to the inclusion and exclusion criteria. Any discrepancies were resolved; when a consensus was not reached, a third researcher was consulted. At level 2 screening, full texts of studies selected at level 1 were obtained and reviewed for eligibility, using the same inclusion and exclusion criteria. Single screening was performed for $52 \%$ of articles; double screening was performed for $48 \%$ of articles. The inclusion and exclusion processes were documented. Only articles published in the English language were reviewed.

Quality of the included studies then was assessed using a modified version of the checklist recommended by the National Institute of Health and Care Excellence (NICE) (18), which is a validated and accepted quality-assessment checklist and which has been used previously to assess study quality for AIT trials (19). Table II presents the items comprising the NICE checklist and the methods used to assess each item. Primary trial publications were the focus of this review; however, any previously published articles describing the study design or methodology of a trial that were cited in the primary publication for that trial also were consulted to identify additional details about the quality-assessment items. Although the quality-assessment items that constitute the NICE checklist are somewhat subjective, they were evaluated consistently across studies, supporting the comparability of the assessments. 
Table I - List of criteria for the inclusion and exclusion of articles.

\begin{tabular}{|c|c|c|}
\hline Criteria & Included & Excluded \\
\hline \multicolumn{3}{|l|}{ Level 1} \\
\hline Study design & $\begin{array}{l}\text { Randomised, double-blind, placebo-controlled trials } \\
\text { Long-term follow-up studies (e.g., open-label follow- } \\
\text { up of randomised, double-blind, placebo-controlled } \\
\text { trials) } \\
\text { Systematic reviews and metaanalyses }{ }^{\mathrm{a}}\end{array}$ & $\begin{array}{l}\text { Nonrandomised studies } \\
\text { Open-label randomised studies } \\
\text { Phase } 1 \text { studies } \\
\text { Proof-of-concept studies } \\
\text { Prognostic studies } \\
\text { Comments } \\
\text { Editorials } \\
\text { Letters } \\
\text { Case reports } \\
\text { Studies in animals but not humans }\end{array}$ \\
\hline Population & $\begin{array}{l}\text { Adults and children with grass pollen AR or ARC } \\
\text { undergoing treatment with AIT }\end{array}$ & $\begin{array}{l}\text { Patients without } \mathrm{AR} \text { or } \mathrm{ARC} \\
\text { Patients with } \mathrm{AR} \text { or ARC induced by allergens other than } \\
\text { grass or grass pollen, e.g., house dust mites, animal dander/ } \\
\text { animal allergens, tree pollen or mould }\end{array}$ \\
\hline Outcomes & No limits & None \\
\hline Language & English & Non-English \\
\hline \multicolumn{3}{|l|}{ Level 2} \\
\hline Outcomes & $\begin{array}{l}\text { Efficacy (AR symptom reduction; AR medication } \\
\text { use reduction; asthma symptom reduction; asthma } \\
\text { medication use reduction) } \\
\text { Safety and tolerability } \\
\text { Quality of life } \\
\text { Compliance } \\
\text { Patient preference }\end{array}$ & Articles that do not report any of the outcomes of interest \\
\hline Language & English & Non-English \\
\hline
\end{tabular}

Abbreviations: AIT, allergy immunotherapy; AR, allergic rhinitis; ARC, allergic rhinitis with conjunctivitis.

Note. Any issues with study design will be reported via the quality-assessment process.

aSystematic reviews and meta-analyses will be used for identification of primary articles. 
Table II - Items assessed in the modified NICE RCT checklist.

\begin{tabular}{ll}
\hline NICE RCT Checklist Item & Response \\
\hline Was randomisation carried out appropriately? & yes/no/not clear/NA \\
\hline Was the concealment of treatment allocation adequate? & yes/no/not clear/NA \\
\hline $\begin{array}{l}\text { Were the groups similar at the outset of the study in terms of prognostic factors [baseline } \\
\text { characteristics]?c }\end{array}$ & yes/no/not clear/NA \\
\hline Were the care providers, participants, and outcome assessors blind to treatment allocation? & yes/no/not clear/NA \\
\hline Were there any unexpected imbalances in dropouts between groups? & yes/no/not clear/NA \\
\hline Is there any evidence to suggest that the authors measured more outcomes than they reported? & yes/no/not clear/NA \\
\hline Were all randomised patients included in the analyses? & yes/no/not clear/NA \\
\hline
\end{tabular}

NA, not applicable; NICE, National Institute of Health and Care Excellence; RCT, randomised controlled trial.

${ }^{a}$ The process of randomisation was found to be appropriate if the authors provided further elaboration on the methods used to generate the random allocation such as a table of random numbers or a computerised random number generator;

${ }^{\mathrm{b}}$ Allocation concealment, which is the method used to implement the random allocation, was sufficient if participants had no prior knowledge of treatment assignment by using an external body, sequentially numbered containers, centralised assignments, or an automated system;

'Prognostic factors of treatment groups were classed as similar where authors reported that there were no significant differences in baseline characteristics or the reported baseline characteristics were similar across groups;

${ }^{\mathrm{d}}$ Blinding was adequate if authors explicitly stated that participants were blinded and described the use of a placebo that was similar to the active drug;

'Dropout rates were considered to be balanced if the proportion of patients withdrawing from the trial were similar across the groups;

fOutcome reporting was considered adequate if authors reported all outcomes stated in the methods section or provided sufficient information about where the additional data could be located:

sThe inclusion of all randomised patients in the analyses was considered adequate if all randomised patients were included in the efficacy and safety analyses. Source: CRD (18).

\section{Results}

Search results

A total of 444 potentially relevant unique records from the January 2017 searches and 50 potentially relevant records from the April 2018 searches were identified for screening: 383 (from January 2017) and 49 (from April 2018) published studies from the database searches, 17 conference abstracts from the Internet searches (January 2017), and 44 (January 2017), and 1 (April 2018) published studies from hand searches of bibliographies. After level 1 screening, 210 (January 2017; databases 162; Internet searches 6; hand searches 42) and 20 (April 2018; databases 19; hand searches 1) studies were progressed for further screening. After level 2 screening, 80 articles were included (databases 61; Internet searches 1; hand searches 18); 44 were unique studies (primary reports) and 36 were secondary reports ( 1 of which was a randomised, double-blind, placebo-controlled study protocol). For the purposes of conducting the quality assessments, all conference abstracts then were excluded because they included insufficient detail about the assessment items (figure 1).

Among the 44 unique studies identified, 17 (38.6\%) were SCIT trials and 27 (61.4\%) were SLIT trials. Overall, 5 trials reported on Allergovit, 8 on Alutard, 2 on Pollinex, and 1 each on Phostal and Purethal (all SCITs); 13 trials reported on Grazax, 6 on Oralair, and 8 on Staloral (all SLITs). These treatments were compared with placebo in all trials. No studies reporting on ALK start or Polvac were identified.

\section{Quality Assessment}

\section{Overall quality}

The results of the quality assessment by year and by treatment type (SCIT vs. SLIT) are shown in figures $\mathbf{2}$ and $\mathbf{3}$, respectively. Overall, 3 of 44 trials $(6.8 \%)$, all Grazax studies, fulfilled every quality criterion in the NICE checklist (figure 3).

The sample sizes of included trials ranged from 18 subjects (20) to 1,501 subjects (21). Trials of Grazax included the largest number of subjects across the included trials $(5,832$ subjects in total), followed by Oralair (2,227 subjects), Alutard ( 830 subjects), Staloral (789 subjects), Allergovit (281 subjects), Pollinex (258 subjects), Purethal (60 subjects), and Phostal (29 subjects). Nineteen trials included $<100$ subjects: 4 of Allergovit, 5 of Alutard, 1 of Phostal, 1 of Purethal, 2 of Grazax, 1 of Oralair, and 5 of Staloral. Ten trials included 100 to 199 subjects: 1 of Allergovit, 2 of Alutard, 2 of Pollinex, 2 of Grazax, 1 of Oralair, and 2 of Staloral. Four trials included 200 to 299 subjects: 2 of Grazax, 1 of Oralair, and 1 of Staloral. Two trials, both of Grazax, included 300 to 399 subjects. Eight trials included 400 to 999 subjects: 1 of Alutard, 4 of Grazax, and 3 of Oralair. One Grazax trial included 1,501 subjects (21). Twenty-seven trials were conducted in adults (Allergovit, 3 trials; Alutard, 6 trials; Grazax, 8 trials; Oralair, 4 trials; Staloral, 2 trials; Phostal, 1 trial; Pollinex, 2 trials, Purethal, 1 trial), 9 included only children (Alutard, 1 trial; Grazax, 4 trials; Oralair, 1 trial; Staloral, 3 trials), and 8 included both children and adults (Allergovit, 2 trials, Alutard, 1 trial; Grazax, 1 trial; Oralair, 1 trial; 
Figure 1 - PRISMA Diagram.

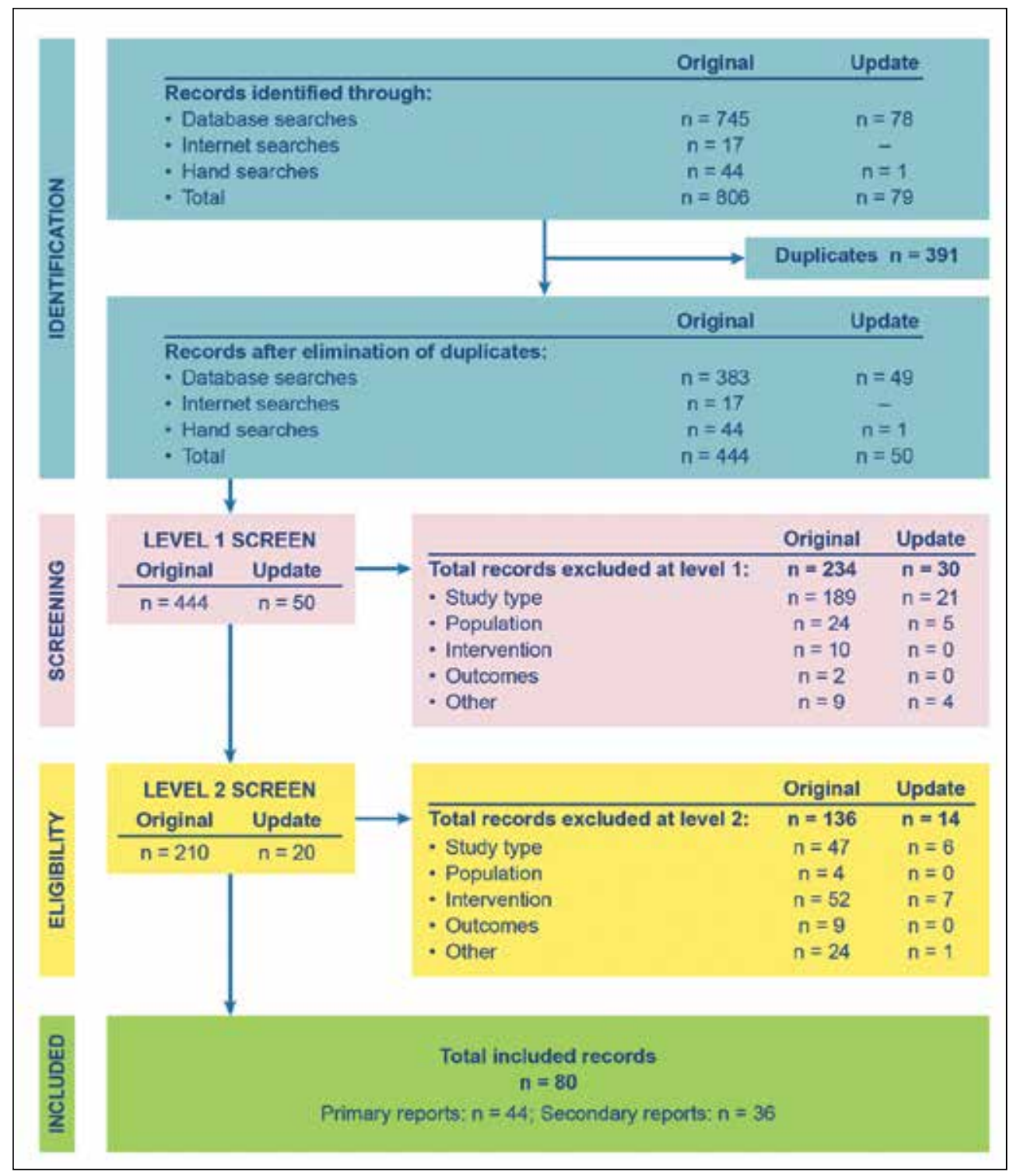

PRISMA, Preferred Reporting Items for Systematic Reviews and Meta-Analyses.

Staloral, 3 trials). One trial conducted in children and 2 trials conducted in adults met all 7 of the quality criteria.

In general, there was a trend towards improved study quality over time: more recent studies, particularly those published after 2009, appropriately addressed more of the quality-assessment items relative to older studies (figure 2). Furthermore, for each product, study sizes tended to increase over time (figure $\mathbf{3}$ ).

\section{Randomisation and concealment of treatment allocation}

The process of randomisation was found to be appropriate if the authors provided further elaboration on the methods used to generate the random allocation (e.g., a table of random numbers or a computerised random number generator). Allocation concealment was considered sufficient if participants had no prior knowledge of treatment assignment by using an external body, sequentially numbered containers, centralised assignments, or an automated system.

Of the 44 included trials, 21 (47.7\%) reported methods of randomisation in detail and $21(47.7 \%)$ adequately described concealment of treatment allocation. By treatment, appropriate randomisation methods were reported for $100 \%$ of Pollinex trials $(2 / 2), 62.5 \%$ of Alutard trials (5/8), $61.5 \%$ of Grazax trials 
Figure 2 - Summary of quality assessment of included randomised controlled trials, by year.

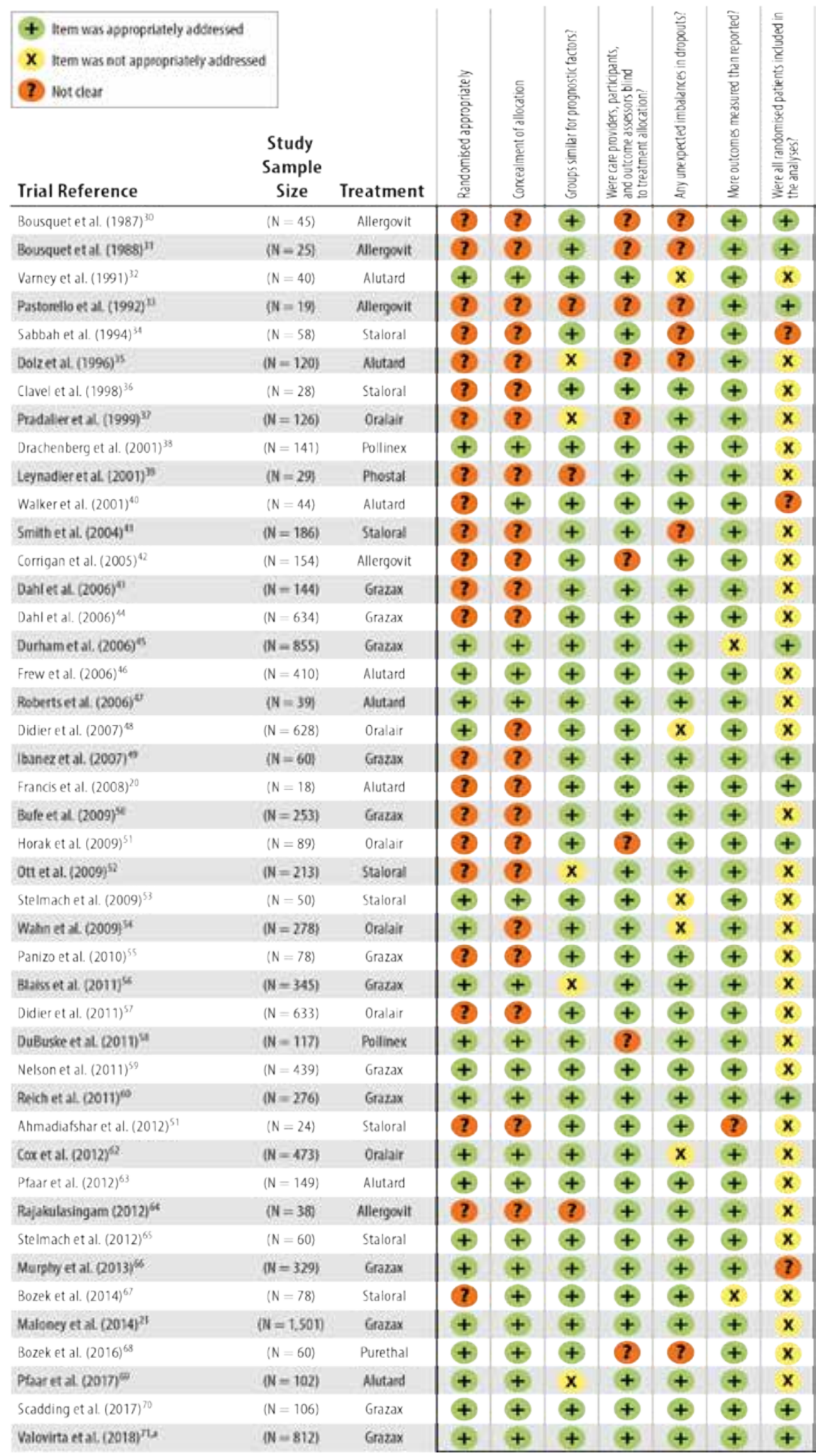

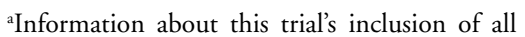
randomised patients in the analyses was presented in Valovirta et al. (72), which was cited as a methods paper in the primary trial publication.

Note. The process of randomisation was found to be appropriate if the authors provided further elaboration on the methods used to generate the random allocation such as a table of random numbers or a computerised random number generator. Allocation concealment, which is the method used to implement the random allocation, was sufficient if participants had no prior knowledge of treatment assignment by using an external body, sequentially numbered contain-ers, centralised assignments, or an automated system. Prognostic factors (or baseline characteris-tics) of treatment groups were classed as similar where authors reported that there were no sig-nificant differences in baseline characteristics or the reported baseline characteristics were simi-lar across groups. Blinding was adequate if authors explicitly stated that participants were blinded and described the use of a placebo that was similar to the active drug. Dropout rates were considered to be balanced if the proportion of patients withdrawing from the trial was sim-ilar across the groups. Outcome reporting was considered adequate if authors reported all out-comes stated in the methods section or provided sufficient information about where the addi-tional data could be located. The inclusion of all randomised patients in the analyses was consid-ered adequate if all randomised patients were included in the efficacy and safety analyses. 
Figure 3 - Summary of quality assessment of included randomised controlled trials, by SCITs vs. SLITs.

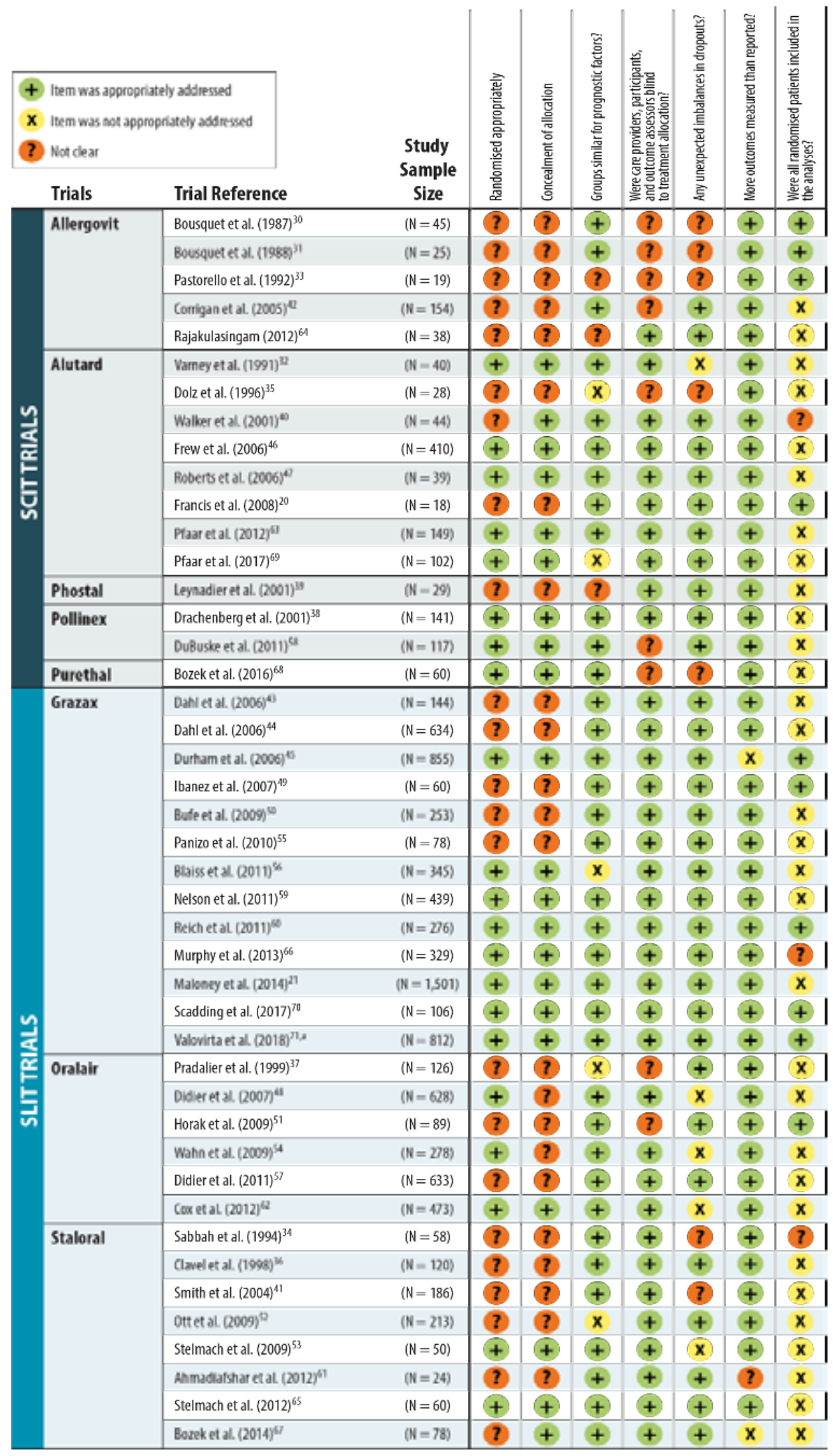

SCIT, subcutaneous immunotherapy; SLIT, sublingual immunotherapy.

anformation about this trial's inclusion of all randomised patients in the analyses was presented in Valovirta et al. (72), which was cited as a methods paper in the primary trial publication.

Note. The process of randomisation was found to be appropriate if the authors provided further elaboration on the methods used to generate the random allocation such as a table of random numbers or a computerised random number generator. Allocation concealment, which is the method used to implement the random allocation, was sufficient if participants had no prior knowledge of treatment assignment by using an external body, sequentially numbered contain-ers, centralised assignments, or an automated system. Prognostic factors (or baseline characteris-tics) of treatment groups were classed as similar where authors reported that there were no sig-nificant differences in baseline characteristics or the reported baseline characteristics were simi-lar across groups. Blinding was adequate if authors explicitly stated that participants were blinded and described the use of a placebo that was similar to the active drug. Dropout rates were considered to be balanced if the proportion of patients withdrawing from the trial was sim-ilar across the groups. Outcome reporting was considered adequate if authors reported all out-comes stated in the methods section or provided sufficient information about where the addi-tional data could be located. The inclusion of all randomised patients in the analyses was consid-ered adequate if all randomised patients were included in the efficacy and safety analyses. 
(8/13), $50.0 \%$ of Oralair trials (3/6), $25.0 \%$ of Staloral trials (2/8), and no trials of Allergovit or Phostal (figure 3). Adequate concealment of treatment allocation was reported for $100 \%$ of Pollinex trials (2/2), $75.0 \%$ of Alutard trials (6/8), 61.5\% of Grazax trials (8/13), 37.5\% of Staloral trials (3/8), $16.7 \%$ of Oralair trials (1/6), and no trials of Allergovit or Phostal. Among SCIT studies, $47.1 \%(8 / 17)$ reported methods of randomisation in detail and $52.9 \%(9 / 17)$ adequately described concealment of treatment allocation; among SLIT trials, 48.1\% (13/27) and $44.4 \%(12 / 27)$, respectively, appropriately addressed these measures. In general, studies published after 2009 more consistently used and/or reported methods of randomisation and allocation concealment than older studies (figure 2).

\section{Similarity of baseline characteristics}

Baseline characteristics of treatment groups were classed as similar where authors reported that there were no significant differences in baseline characteristics or the reported baseline characteristics were similar across groups.

Overall, in 37 of 44 trials (84.1\%), treatment and placebo groups had similar baseline characteristics. Baseline characteristics were similar between groups in a majority of trials for each treatment: Pollinex, $100 \%$ of trials (2/2); Purethal, $100 \%$ (1/1); Grazax, 92.3\% (12/13); Staloral, 87.5\% (7/8); Oralair, 83.3\% (5/6); Alutard, 75.0\% (6/8); Allergovit, 60\% (3/5); and Phostal, 0. Among SCIT trials, 70.6\% (12/17) had treatment groups with similar baseline characteristics, whereas $88.9 \%$ of SLIT trials $(24 / 27)$ had treatment groups with similar baseline characteristics.

\section{Blinding of treatment allocation}

Blinding of treatment allocation was considered adequate if authors explicitly stated that participants were blinded and described the use of a placebo that was similar to the active drug. Although all trials were reported to be double blind, it was unclear whether subjects were blinded appropriately in 9 of 44 trials overall $(20.5 \%)$. By treatment, $100 \%$ of Grazax trials (13/13), $100 \%$ of Staloral trials (8/8), $87.5 \%$ of Alutard trials (7/8), $66.7 \%$ of Oralair trials (4/6), 50\% of the Pollinex trials (1/2), 20\% of Allergovit trials (1/5), and the Phostal trial clearly reported on blinding procedures, whereas the Purethal trial did not. More SLIT trials $(92.6 \%, 25 / 27)$ than SCIT trials $(58.8 \%, 10 / 17)$ clearly reported on blinding procedures.

\section{Unexpected imbalances in dropouts}

Dropout rates were considered to be balanced if the proportion of patients withdrawing from the trial was similar across treatment groups.

In 5 of 44 trials $(11.4 \%)$, there were unexpected imbalances in dropouts between treatment groups, and this item was not clearly reported in 7 trials $(15.9 \%)$. By treatment, $60.0 \%$ of Allergovit trials (3/5), $37.5 \%$ of Staloral trials (3/8), $50.0 \%$ of
Oralair trials (3/6), 25.0\% of Alutard trials (2/8), and the Purethal trial either included or did not clearly report on imbalances in dropouts. No such imbalances were included in any of the 13 Grazax trials, the 2 Pollinex trials, or the Phostal trial. Proportionally more SCIT trials $(35.3 \%, 6 / 17)$ than SLIT trials $(22.2 \%, 6 / 27)$ included or did not clearly report on imbalances in dropouts.

\section{Evidence of outcomes assessed and not reported}

Outcome reporting was considered adequate if authors reported all outcomes stated in the methods section or provided sufficient information about where the additional data could be located.

Overall, 2 of 44 trials (4.5\%) did not report all outcomes assessed, and this was unclear in 1 trial (2.3\%). All trials of Allergovit (5/5), Alutard (8/8), Pollinex (2/2), Oralair (6/6), and Phostal and Purethal (1 each) reported on all outcomes assessed, whereas $92.3 \%$ of Grazax trials (12/13) and $75.0 \%$ of Staloral trials (6/8) reported on all outcomes assessed. All 17 SCIT trials and $88.9 \%$ of SLIT trials (24/27) reported on all outcomes assessed.

\section{Inclusion of all randomised patients in the analyses}

The inclusion of all randomised patients in the analyses was considered adequate if all randomised patients were included in the efficacy and safety analyses.

Overall, 10 of 44 trials (20.7\%) included all randomised patients in the analyses. By treatment, $60 \%$ of Allergovit trials (3/5), 38.5\% of Grazax trials (5/13), $16.7 \%$ of Oralair trials $(1 / 6)$, and $12.5 \%$ of Alutard trials (1/8) included an all randomised patients in the analyses; none of the Staloral, Pollinex, Phostal, or Purethal trials included all randomised patitents in the analyses. Such analyses were included in $23.5 \%$ of SCIT trials (4/17) and $22.2 \%$ of SLIT trials (6/27).

\section{Discussion}

In this systematic literature review to assess the quality of 44 placebo-controlled trials of grass allergy AITs, only 3 trials, all Grazax studies, fulfilled all quality criteria in the modified NICE checklist. Consistent with previous findings that more recently conducted AIT trials are of better quality than older trials (22), our review found that more recent trials (published after 2009) were generally of better quality and reporting than older trials, both overall and for individual products. The trend towards improved quality over time is potentially a reflection of evolving standards for both trial design and reporting. More recent studies also tended to include larger sample sizes relative to older studies, both overall and for the individual products. Notably, 5 Grazax trials, 3 Oralair trials, and 1 Alutard trial each enrolled more than 400 subjects, and 1 of these Grazax trials included 1,501 subjects. Trials of SLIT products generally included larger sample sizes than SCIT trials. 
Inconsistencies in the quality and reporting of trial methods were revealed when quality assessments were performed. Several trials reported that they were randomised, but only a few reported the methods used for randomisation, concealment of treatment allocation, inclusion of all randomised patients in the analyses, or handling of missing data. In particular, studies published after 2009 more consistently addressed randomisation and concealment of treatment allocation compared with older trials. In most trials, treatment groups had similar baseline characteristics. Although all trials were double blind, it was unclear whether blinding procedures were appropriate in approximately $20 \%$ of trials overall; more SLIT trials than SCIT trials clearly reported on blinding methods. Few trials overall, and proportionally more SCIT trials than SLIT trials, reported imbalances in dropouts between treatment groups. All SCIT trials and all but 3 SLIT trials reported on all outcomes assessed. Only a quarter of trials overall, and similar proportions of SCIT trials (approximately 24\%) and SLIT trials (approximately 22\%), included all randomised patients in the analyses. When studies were compared across the treatments reviewed, Grazax studies were of high quality relative to trials of other treatments, according to the quality assessments. Specifically, all Grazax trials included appropriate blinding methods and avoided unexpected imbalances in dropouts. In addition, $92 \%$ of Grazax trials reported on all outcomes assessed. Three Grazax trials appropriately addressed all 7 quality criteria. Although fewer trials of Alutard than of Grazax were identified (8 vs. 13), Alutard trials were also of good quality overall, with $87.5 \%$ of trials appropriately addressing 5 or more quality criteria.

The quality-assessment results from this study provide important context for the assessment of clinical endpoints and other outcomes in AIT. For example, previous research has explored the effects of SCIT versus SLIT for respiratory allergy. Although both SCIT and SLIT have been shown to be effective, SCIT is associated with a higher risk of life-threatening systemic reactions than SLIT (23). In contrast with SCIT, SLIT is suitable for at-home administration, is less painful and more convenient owing to a lack of injection, has a lower risk of anaphylaxis, has lower indirect costs, and has been shown to be cost saving relative to pharmacotherapy (15,24-26).

Physicians, patients, and payers considering AIT options for respiratory allergy should consider not only the attributes and outcomes of available treatments but also the robustness of the underlying evidence. Given the unique regulatory history of AIT products in Europe, some products have been registered or are in use that lack a solid evidence base. Nevertheless, evidentiary standards for AIT products are evolving, as can be observed from the AIT landscape in Germany. Guidelines on the use of AIT issued jointly by German, Austrian, and Swiss professional organizations in 2014 acknowledge that data from SCIT and SLIT trials differ in quality and scope and recommend prod- uct-specific evaluations to inform treatment decisions (27). In conjunction with these guidelines, a summary of the currently available AIT products (including registration dates) and a separate summary of the evidence fulfilling defined quality criteria, including study quality, supporting the available products are issued every 6 months $(28,29)$. The intent of these resources is to enhance transparency for AIT products to support the physicians in their guidelines-based therapy decisions. Whether other health care systems will adopt a similar focus on quality of evidence for AIT products remains to be seen.

Some strengths and limitations of this study must be considered when the results are interpreted. Studies were identified systematically using a comprehensive search strategy with no date limitations and were screened according to predefined inclusion and exclusion criteria. Study quality was assessed using an accepted, validated measure that has been used previously to assess the quality of AIT evidence (19). Nevertheless, there are some limitations associated with the quality-assessment method in that studies indicating that they fulfilled a particular quality-assessment item (e.g., randomisation) but did not clearly describe the methods used for that item were classified as "not clear." Such classifications reflect incomplete reporting of the trial, potentially in line with reporting standards that were in place when the trial was published, and not necessarily poor quality. In particular, 5 trials ( 3 of Allergovit, 1 of Alutard, and 1 of Staloral) were published before the first CONSORT statement was issued in 1996. Finally, only articles published in the English language were reviewed, and thus trials published in other languages are not reflected in the results.

\section{Conclusions}

Considering the historical perspective on and the evolving evidentiary standards for AIT trials, it is important to understand the quality of the existing clinical evidence. Although the results here are only for grass AIT, it is likely that similar results would be found for other AIT products. The marketing and use of AIT products in Europe and worldwide are heterogeneous and historically have been guided by expert clinical opinion rather than close regulatory oversight (9). The standards for clinical evidence for AITs have evolved in recent decades, however; accordingly, the quality of AIT trials has tended to improve over time, with more recent trials generally including higher numbers of patients and appropriately addressing more quality-assessment items than older studies. This SLR focused on the published data for registered grass AIT. Published evidence is of better quality or is more extensive for some of the reviewed treatments than others. In particular, numerous trials have been published for Grazax and Alutard, which were of good quality on the whole. In comparison, evidence was limited for Phostal and Purethal. Our results support previous recommendations 
that future trials in AIT should be robustly designed, in line with accepted quality metrics, and should consistently and completely report findings to aid their appraisal and interpretation $(17,27)$. In particular, trials should use appropriate methods for randomisation, allocation concealment, blinding, inclusion of all randomised patients in the analyses, and accounting for missing data; should ensure balance between treatment groups in baseline characteristics and report on unexpected imbalances in dropouts; and should ensure reporting of all outcomes assessed. Evidence-based treatment decisions for AITs should rely on not only trial outcomes but also the quality of the evidence base.

\section{Conflict of interest}

Annete Njue, Weyinmi Nuabor, Matthew Lyall, and Anne Heyes are salaried employees of RTI Health Solutions. Lisa Elliott is a salaried employee of ALK-Abelló. Anne Domdey was a salaried employee of ALK-Abelló when this research was conducted.

\section{Funding}

This study was conducted under a research contract between RTI Health Solutions and ALK-Abelló and was funded by ALK-Abelló.

\section{Acknowledgements}

Mary Levine of RTI Health Solutions provided overall project management for this study. Kate Lothman of RTI Health Solutions provided medical-writing assistance, which was funded by ALK-Abelló.

\section{Author contributions}

Anne Domdey initiated the study, secured funding, designed the study, interpreted the data, and participated in writing the manuscript. Annete Njue designed the study, screened the articles, led and conducted the analyses, interpreted the data, and participated in writing the manuscript. Weyinmi Nuabor and Matthew Lyall screened the articles, conducted the analyses, interpreted the data, and participated in writing the manuscript. Anne Heyes provided study oversight, contributed to the study design and analyses, interpreted the data, and participated in writing the manuscript. Lisa Elliott secured funding, contributed to the study design, interpreted the data, and participated in writing the manuscript.

\section{References}

1. Fiocchi A, Fox AT. Preventing progression of allergic rhinitis: the role of specific immunotherapy. Arch Dis Child Educ Pract Ed 2011; 96(3):91-100.
2. Feng $\mathrm{CH}$, Miller MD, Simon RA. The united allergic airway: connections between allergic rhinitis, asthma, and chronic sinusitis. Am J Rhinol Allergy 2012; 26(3):187-190.

3. EFA. EFA book on respiratory allergies. European Federation of Allergy and Airways Diseases Patients Association; 2011. Available at: http://www.eaaci.org/resources/educational-tools/ books/1864-efa-book-on-respiratory-allergies.html. Accessed 4 March 2016.

4. Scadding G, Walker S. Poor asthma control?--then look up the nose. The importance of co-morbid rhinitis in patients with asthma. Prim Care Respir J 2012; 21(2):222-228.

5. Burgess JA, Walters EH, Byrnes GB, Matheson MC, Jenkins MA, Wharton CL, et al. Childhood allergic rhinitis predicts asthma incidence and persistence to middle age: a longitudinal study. J Allergy Clin Immunol 2007; 120(4):863-869.

6. Morjaria JB, Caruso M, Rosalia E, Russo C, Polosa R. Preventing progression of allergic rhinitis to asthma. Curr Allergy Asthma Rep 2014; 14(2):412.

7. Poddighe D, Licari A, Caimmi S, Marseglia GL. Sublingual immunotherapy for pediatric allergic rhinitis: the clinical evidence. World J Clin Pediatr 2016; 5(1):47-56.

8. Bousquet J, Khaltaev N, Cruz AA, Denburg J, Fokkens WJ, Togias A, et al. Allergic Rhinitis and Its Impact on Asthma (ARIA) 2008 update (in collaboration with the World Health Organization, GA(2)LEN and AllerGen). Allergy 2008; 63 Suppl 86:8-160.

9. Bonertz A, Roberts GC, Hoefnagel M, Timon M, Slater JE, Rabin $\mathrm{RL}$, et al. Challenges in the implementation of EAACI guidelines on allergen immunotherapy: a global perspective on the regulation of allergen products. Allergy 2018; 73(1):64-76.

10. Kaul S, May S, Luttkopf D, Vieths S. Regulatory environment for allergen-specific immunotherapy. Allergy 2011; 66(6):753-764.

11. Larsen JN, Broge L, Jacobi H. Allergy immunotherapy: the future of allergy treatment. Drug Discov Today 2016; 21(1):26-37.

12. Bonini S. Regulatory aspects of allergen-specific immunotherapy: Europe sets the scene for a global approach. World Allergy Organ J 2012; 5(10):120-123.

13. McDonell AL, Wahn U, Demuth D, Richards C, Hawes C, Andreasen JN, et al. Allergy immunotherapy prescribing trends for grass pollen-induced allergic rhinitis in Germany: a retrospective cohort analysis. Allergy Asthma Clin Immunol 2015; 11(1):19.

14. Bachert C, Larche M, Bonini S, Canonica GW, Kundig T, Larenas-Linnemann $\mathrm{D}$, et al. Allergen immunotherapy on the way to product-based evaluation-a WAO statement. World Allergy Organ J 2015; 8(1):29.

15. Makatsori M, Calderon MA. Sublingual allergen immunotherapy for respiratory allergies: what is new? Expert Rev Clin Immunol 2014; 10(12):1641-1647.

16. Brozek JL, Bousquet J, Baena-Cagnani CE, Bonini S, Canonica GW, Casale TB, et al. Allergic Rhinitis and Its Impact on Asthma (ARIA) guidelines: 2010 revision. J Allergy Clin Immunol 2010; 126(3):466-476.

17. Roberts G, Pfaar O, Akdis CA, Ansotegui IJ, Durham SR, Gerth van Wijk R, et al. EAACI guidelines on allergen immunotherapy: allergic rhinoconjunctivitis. Allergy 2018; 73(4):765-798.

18. CRD. CRD's guidance for undertaking reviews in health care. 2008. Available at: https://www.york.ac.uk/media/crd/Systematic_Reviews.pdf. Accessed 30 April 2018.

19. Nelson H, Cartier S, Allen-Ramey F, Lawton S, Calderon MA. Network meta-analysis shows commercialized subcutaneous and 
sublingual grass products have comparable efficacy. J Allergy Clin Immunol Pract 2015; 3(2):256-266.e3.

20. Francis JN, James LK, Paraskevopoulos G, Wong C, Calderon MA, Durham SR, et al. Grass pollen immunotherapy: IL-10 induction and suppression of late responses precedes IgG4 inhibitory antibody activity. J Allergy Clin Immunol 2008; 121(5):1120-5.e2.

21. Maloney J, Bernstein DI, Nelson H, Creticos P, Hebert J, Noonan $\mathrm{M}$, et al. Efficacy and safety of grass sublingual immunotherapy tablet, MK-7243: a large randomized controlled trial. Ann Allergy Asthma Immunol 2014; 112(2):146-153 e2.

22. Bousquet PJ, Calderon MA, Demoly P, Larenas D, Passalacqua G, Bachert C, et al. The Consolidated Standards of Reporting Trials (CONSORT) Statement applied to allergen-specific immunotherapy with inhalant allergens: a Global Allergy and Asthma European Network (GA(2)LEN) article. J Allergy Clin Immunol 2011; 127(1):49-56,e1-11.

23. Incorvaia C, Mauro M, Ridolo E. Sublingual immunotherapy for allergic rhinitis: where are we now? Immunotherapy 2015; 7(10):1105-1110.

24. Vadlamudi A, Shaker M. New developments in allergen immunotherapy. Curr Opin Pediatr 2015; 27(5):649-655.

25. Ronborg SM, Svendsen UG, Micheelsen JS, Ytte L, Andreasen JN, Ehlers L. Budget impact analysis of two immunotherapy products for treatment of grass pollen-induced allergic rhinoconjunctivitis. Clinicoecon Outcomes Res 2012; 4:253-260.

26. Domdey A, Grand TS, Elliot L, Tesch F, Schmitt J, Küster D. Costs and resource use in allergic rhinitis. Presented at the European Academy of Allergy and Clinical Immunology; 26-30 May 2018. Munich, Germany.

27. Pfaar O, Bachert C, Bufe A, Buhl R, Ebner C, Eng P, et al. Guideline on allergen-specific immunotherapy in IgE-mediated allergic diseases: S2k Guideline of the German Society for Allergology and Clinical Immunology (DGAKI), the Society for Pediatric Allergy and Environmental Medicine (GPA), the Medical Association of German Allergologists (AeDA), the Austrian Society for Allergy and Immunology (OGAI), the Swiss Society for Allergy and Immunology (SGAI), the German Society of Dermatology (DDG), the German Society of Oto- Rhino-Laryngology, Head and Neck Surgery (DGHNO-KHC), the German Society of Pediatrics and Adolescent Medicine (DGKJ), the Society for Pediatric Pneumology (GPP), the German Respiratory Society (DGP), the German Association of ENT Surgeons (BV-HNO), the Professional Federation of Paediatricians and Youth Doctors (BVKJ), the Federal Association of Pulmonologists (BDP) and the German Dermatologists Association (BVDD). Allergo J Int 2014; 23(8):282-319.

28. German Society for Allergology and Clinical Immunology. Präparate zur spez. Immuntherapie (D, 12-2018). December 2018. Available at: http://www.dgaki.de/wp-content/uploads/2014/11/ SIT-Produkte_Studien_Zulassung_01-2017.pdf Accessed 18 March 2019.

29. German Society for Allergology and Clinical Immunology. Studien mit positivem Wirksamkeitsnachweis - Gräserpollen 12-2018. 2018. Available at: https://www.dgaki.de/leitlinien/s2k-leitliniesit/. Accessed 18 March 2019.

30. Bousquet J, Hejjaoui A, Skassa-Brociek W, Guerin B, Maasch HJ, Dhivert H, et al. Double-blind, placebo-controlled immunotherapy with mixed grass-pollen allergoids. I. Rush immunotherapy with allergoids and standardized orchard grass-pollen extract. J Allergy Clin Immunol 1987; 80(4):591-598.
31. Bousquet J, Maasch H, Martinot B, Hejjaoui A, Wahl R, Michel FB. Double-blind, placebo-controlled immunotherapy with mixed grass-pollen allergoids. II. Comparison between parameters assessing the efficacy of immunotherapy. J Allergy Clin Immunol 1988; 82(3 Pt 1):439-446.

32. Varney VA, Gaga M, Frew AJ, Aber VR, Kay AB, Durham SR. Usefulness of immunotherapy in patients with severe summer hay fever uncontrolled by antiallergic drugs. BMJ 1991; 302(6771):265269.

33. Pastorello EA, Pravettoni V, Incorvaia C, Mambretti M, Franck E, Wahl R, et al. Clinical and immunological effects of immunotherapy with alum-absorbed grass allergoid in grass-pollen-induced hay fever. Allergy 1992; $47(4$ Pt 1):281-290.

34. Sabbah A, Hassoun S, Le Sellin J, Andre C, Sicard H. A double-blind, placebo-controlled trial by the sublingual route of immunotherapy with a standardized grass pollen extract. Allergy 1994; 49(5):309-313.

35. Dolz I, Martinez-Cocera C, Bartolome JM, Cimarra M. A double-blind, placebo-controlled study of immunotherapy with grass-pollen extract Alutard SQ during a 3-year period with initial rush immunotherapy. Allergy 1996; 51(7):489-500.

36. Clavel R, Bousquet J, Andre C. Clinical efficacy of sublingual-swallow immunotherapy: a double-blind, placebo-controlled trial of a standardized five-grass-pollen extract in rhinitis. Allergy 1998; 53(5):493-498.

37. Pradalier A, Basset D, Claudel A, Couturier P, Wessel F, Galvain $S$, et al. Sublingual-swallow immunotherapy (SLIT) with a standardized five-grass-pollen extract (drops and sublingual tablets) versus placebo in seasonal rhinitis. Allergy 1999; 54(8):819-828.

38. Drachenberg KJ, Wheeler AW, Stuebner P, Horak F. A well-tolerated grass pollen-specific allergy vaccine containing a novel adjuvant, monophosphoryl lipid A, reduces allergic symptoms after only four preseasonal injections. Allergy 2001; 56(6):498-505.

39. Leynadier F, Banoun L, Dollois B, Terrier P, Epstein M, Guinnepain MT, et al. Immunotherapy with a calcium phosphate-adsorbed five-grass-pollen extract in seasonal rhinoconjunctivitis: a double-blind, placebo-controlled study. Clin Exp Allergy 2001; 31(7):988-996.

40. Walker SM, Pajno GB, Lima MT, Wilson DR, Durham SR. Grass pollen immunotherapy for seasonal rhinitis and asthma: a randomized, controlled trial. J Allergy Clin Immunol 2001; 107(1):87-93.

41. Smith H, White P, Annila I, Poole J, Andre C, Frew A. Randomized controlled trial of high-dose sublingual immunotherapy to treat seasonal allergic rhinitis. J Allergy Clin Immunol 2004; 114(4):831-837.

42. Corrigan CJ, Kettner J, Doemer C, Cromwell O, Narkus A, Study G. Efficacy and safety of preseasonal-specific immunotherapy with an aluminium-adsorbed six-grass pollen allergoid. Allergy 2005; 60(6):801-807.

43. Dahl R, Stender A, Rak S. Specific immunotherapy with SQ standardized grass allergen tablets in asthmatics with rhinoconjunctivitis. Allergy 2006; 61(2):185-190.

44. Dahl R, Kapp A, Colombo G, de Monchy JG, Rak S, Emminger W, et al. Efficacy and safety of sublingual immunotherapy with grass allergen tablets for seasonal allergic rhinoconjunctivitis. J Allergy Clin Immunol 2006; 118(2):434-440.

45. Durham SR, Yang WH, Pedersen MR, Johansen N, Rak S. Sublingual immunotherapy with once-daily grass allergen tablets: a ran- 
A. Domdey, A. Njue, W. Nuabor, M. Lyall, A. Heyes, L. Elliott

domized controlled trial in seasonal allergic rhinoconjunctivitis. J Allergy Clin Immunol 2006; 117(4):802-809.

46. Frew AJ, Powell RJ, Corrigan CJ, Durham SR, Group UKIS. Efficacy and safety of specific immunotherapy with SQ allergen extract in treatment-resistant seasonal allergic rhinoconjunctivitis. J Allergy Clin Immunol 2006; 117(2):319-325.

47. Roberts G, Hurley C, Turcanu V, Lack G. Grass pollen immunotherapy as an effective therapy for childhood seasonal allergic asthma. J Allergy Clin Immunol 2006; 117(2):263-268.

48. Didier A, Malling HJ, Worm M, Horak F, Jager S, Montagut A, et al. Optimal dose, efficacy, and safety of once-daily sublingual immunotherapy with a 5-grass pollen tablet for seasonal allergic rhinitis. J Allergy Clin Immunol 2007; 120(6):1338-1345.

49. Ibanez MD, Kaiser F, Knecht R, Armentia A, Schopfer H, Tholstrup B, et al. Safety of specific sublingual immunotherapy with SQ standardized grass allergen tablets in children. Pediatr Allergy Immunol 2007; 18(6):516-522.

50. Bufe A, Eberle P, Franke-Beckmann E, Funck J, Kimmig M, Klimek L, et al. Safety and efficacy in children of an SQ-standardized grass allergen tablet for sublingual immunotherapy. J Allergy Clin Immunol 2009; 123(1):167-173.e7.

51. Horak F, Zieglmayer P, Zieglmayer R, Lemell P, Devillier P, Montagut A, et al. Early onset of action of a 5-grass-pollen 300-IR sublingual immunotherapy tablet evaluated in an allergen challenge chamber. J Allergy Clin Immunol 2009; 124(3):471-477, 7.e1.

52. Ott H, Sieber J, Brehler R, Folster-Holst R, Kapp A, Klimek L, et al. Efficacy of grass pollen sublingual immunotherapy for three consecutive seasons and after cessation of treatment: the ECRIT study. Allergy 2009; 64(9):1394-1401.

53. Stelmach I, Kaczmarek-Woźniak J, Majak P, Olszowiec-Chlebna $\mathrm{M}$, Jerzynska J. Efficacy and safety of high-doses sublingual immunotherapy in ultra-rush scheme in children allergic to grass pollen. Clin Exp Allergy 2009; 39(3):401-408.

54. Wahn U, Tabar A, Kuna P, Halken S, Montagut A, de Beaumont $\mathrm{O}$, et al. Efficacy and safety of 5-grass-pollen sublingual immunotherapy tablets in pediatric allergic rhinoconjunctivitis. J Allergy Clin Immunol 2009; 123(1):160-166 e3.

55. Panizo C, Cimarra M, Gonzalez-Mancebo E, Vega A, Senent C, Martin S. In vivo and in vitro immunological changes induced by a short course of grass allergy immunotherapy tablets. J Investig Allergol Clin Immunol 2010; 20(6):454-462.

56. Blaiss M, Maloney J, Nolte H, Gawchik S, Yao R, Skoner DP. Efficacy and safety of timothy grass allergy immunotherapy tablets in North American children and adolescents. J Allergy Clin Immunol 2011; 127(1):64-71.

57. Didier A, Worm M, Horak F, Sussman G, de Beaumont O, Le Gall M, et al. Sustained 3-year efficacy of pre- and coseasonal 5-grass-pollen sublingual immunotherapy tablets in patients with grass pollen-induced rhinoconjunctivitis. J Allergy Clin Immunol 2011; 128(3):559-566.

58. DuBuske LM, Frew AJ, Horak F, Keith PK, Corrigan CJ, Aberer W, et al. Ultrashort-specific immunotherapy successfully treats seasonal allergic rhinoconjunctivitis to grass pollen. Allergy Asthma Proc 2011; 32(3):239-247.

59. Nelson HS, Nolte H, Creticos P, Maloney J, Wu J, Bernstein DI. Efficacy and safety of timothy grass allergy immunotherapy tablet treatment in North American adults. J Allergy Clin Immunol 2011; 127(1):72-80, .e1-2.

60. Reich K, Gessner C, Kroker A, Schwab JA, Pohl W, Villesen H, et al. Immunologic effects and tolerability profile of in-season initia- tion of a standardized-quality grass allergy immunotherapy tablet: a phase III, multicenter, randomized, double-blind, placebo-controlled trial in adults with grass pollen-induced rhinoconjunctivitis. Clin Ther 2011; 33(7):828-840.

61. Ahmadiafshar A, Maarefvand M, Taymourzade B, Mazloomzadeh S, Torabi Z. Efficacy of sublingual swallow immunotherapy in children with rye grass pollen allergic rhinitis: a double-blind placebo-controlled study. Iran J Allergy Asthma Immunol 2012; 11(2):175-181.

62. Cox L, Casale T, Nayak A, Bernstein D, Creticos P, Mekhaldi S, et al. Efficacy and safety of 300IR 5-grass pollen sublingual allergen immunotherapy tablets in us adults with grass-pollen allergic rhinoconjunctivitis. World Allergy Organ J 2012; 5(Suppl 2):S53-S54

63. Pfaar O, Urry Z, Robinson DS, Sager A, Richards D, Hawrylowicz $\mathrm{CM}$, et al. A randomized placebo-controlled trial of rush preseasonal depigmented polymerized grass pollen immunotherapy. Allergy 2012; 67(2):272-279.

64. Rajakulasingam K. Early improvement of patients' condition during allergen-specific subcutaneous immunotherapy with a high-dose hypoallergenic 6-grass pollen preparation. Eur Ann Allergy Clin Immunol 2012; 44(3):128-134.

65. Stelmach I, Kaluzinska-Parzyszek I, Jerzynska J, Stelmach P, Stelmach W, Majak P. Comparative effect of pre-coseasonal and continuous grass sublingual immunotherapy in children. Allergy 2012; 67(3):312-320.

66. Murphy K, Gawchik S, Bernstein D, Andersen J, Pedersen MR. A phase 3 trial assessing the efficacy and safety of grass allergy immunotherapy tablet in subjects with grass pollen-induced allergic rhinitis with or without conjunctivitis, with or without asthma. J Negat Results Biomed 2013; 12:10.

67. Bozek A, Kolodziejczyk K, Warkocka-Szoltysek B, Jarzab J. Grass pollen sublingual immunotherapy: a double-blind, placebo-controlled study in elderly patients with seasonal allergic rhinitis. Am J Rhinol Allergy 2014; 28(5):423-427.

68. Bozek A, Kolodziejczyk K, Krajewska-Wojtys A, Jarzab J. Pre-seasonal, subcutaneous immunotherapy: a double-blinded, placebo-controlled study in elderly patients with an allergy to grass. Ann Allergy Asthma Immunol 2016; 116(2):156-161.

69. Pfaar O, Hohlfeld JM, Al-Kadah B, Hauswald B, Homey B, Hunzelmann N, et al. Dose-response relationship of a new Timothy grass pollen allergoid in comparison with a 6 -grass pollen allergoid. Clin Exp Allergy 2017; 47(11):1445-1455.

70. Scadding GW, Calderon MA, Shamji MH, Eifan AO, Penagos M, Dumitru F, et al. Effect of 2 years of treatment with sublingual grass pollen immunotherapy on nasal response to allergen challenge at 3 years among patients with moderate to severe seasonal allergic rhinitis: the GRASS randomized clinical trial. JAMA 2017; 317(6):615-625.

71. Valovirta E, Petersen TH, Piotrowska T, Laursen MK, Andersen JS, Sorensen HF, et al. Results from the 5-year SQ grass sublingual immunotherapy tablet asthma prevention (GAP) trial in children with grass pollen allergy. J Allergy Clin Immunol 2018; 141(2):529-538.313.

72. Valovirta E, Berstad AK, de Blic J, Bufe A, Eng P, Halken S, et al. Design and recruitment for the GAP trial, investigating the preventive effect on asthma development of an SQ-standardized grass allergy immunotherapy tablet in children with grass pollen-induced allergic rhinoconjunctivitis. Clin Ther 2011; 33(10):1537-1546. 